\title{
Role of attractive forces in determining the equilibrium structure and dynamics of simple liquids
}

\author{
S. Toxvaerd \\ DNRF centre "Glass and Time", IMFUFA, Department of Sciences, Roskilde University, \\ Postbox 260, DK-4000 Roskilde, Denmark
}

Received July 31, 2014, in final form October 2, 2014

\begin{abstract}
Molecular Dynamics simulations of a Lennard-Jones system with different range of attraction show that the attractive forces modify the radial distribution of the particles. For condensed liquids only, the forces within the the first coordination shell (FCS) are important, but for gases and moderate condensed fluids, even the attractive forces outside the FCS play a role. The changes in the distribution caused by neglecting the attractive forces, lead to a too high pressure. The weak long-range attractions damp the dynamics and the diffusion of the particles in gas-, super critical fluid- and in liquid states. The values of self-diffusion coefficients (SDC) agree qualitatively with a modified Cohen-Turnbull model. The SDC-s along the critical isotherm show no anomaly at the critical point in agreement with experimental data.
\end{abstract}

Key words: simple liquids, equation of state, perturbation theory, self-diffusion

PACS: 05.20.Jj, 47.11.Mn, 64.70.pm, 65.20.De, 66.10.cg, 82.20.Wt

\section{Introduction}

Ever since van der Waals (vdW) [1] in 1873 formulated his theory "the continuity of the gaseous and liquid states”, the understanding of simple fluids has been that repulsive and attractive forces give rise to separate modifications of the ideal-gas equation of state and to separate contributions to the free energy. The harsh, repulsive forces reduce the available volume and determine the structure of the fluid and thus its configurational entropy; the weaker, longer-range attractive forces give rise to an energy effect that reduces pressure and energy compared to those of an ideal gas at the same temperature and density. With respect to the effect of the longer-range attractive forces, van der Waals imagined that he could (quoting from Ref. [2]) “... define an element of volume in a liquid which is small compared ...” with "...the range of the intermolecular force, but large enough for it to contain sufficient molecules for us to assume that there is within it a uniform distribution of molecules of number density, $\rho=n / V^{\prime}$. The pressure $p(n / V, T)$ is in accordance with van der Waals

$$
\left(p+\frac{n^{2} a}{V^{2}}\right)(V-n b)=n R T .
$$

He assumed that the main effect of the attractive forces originates from the molecules within this local sphere and estimated this sphere to have a range of 3-6 $\AA$ [2]. For condensed liquids, the sphere can be identified as the first coordination shell [3].

It is well known that the vdW equation of state reproduces the qualitative behavior of the fluid state [4, 5]. Van der Waals' idea about the role of the repulsive and attractive forces lies behind Zwanzig's hightemperature expansion [6], in which the contribution to the free energy from the long-range forces is expressed in powers of the inverse temperature, the reference high-temperature system being a system with infinitely strong, purely repulsive forces. The success of this perturbation theory (PT) was demonstrated by Longuet-Higgens and Widom [7], Barker and Henderson [8], and soon after by Weeks, Chandler, and Andersen (WCA) in their seminal paper "Role of repulsive forces in determining the equilibrium structure of simple liquids” [9]. 
In PT, a fundamental problem is how to separate the strong repulsive attractions from the weaker long-range attractions. One possible separation was proposed by Barker and Henderson, who marked the separation at the distance where the potential is zero. WCA demonstrated, however, that by choosing instead the separation at the potential minimum, one obtains a much better agreement between the particle distributions of the system and the reference system. Doing so, the forces are separated into purely repulsive and purely attractive forces, and one may say that the original idea of van der Waals is here captured in its purest form. Numerous refinements of perturbation theory have appeared since then. An excellent summary of perturbation approaches until 1976 can be found in Barker and Henderson's classic review [10].

Whereas the vdW equation is used for simple systems in gas-, liquid- and coexisting liquid-gas states, the PT theories are normally only used for condensed liquid states. In WCA [9], only fluid densities $\rho \geqslant 0.65$ were included in the investigation, corresponding to the densities more than twice the critical density. Here, we shall determine the effect of the attractions in a simple Lennard-Jones system on the structure (section 3) and dynamics (section 4), as well as on gas-, liquid- and super-critical fluid states.

\section{Perturbation theory for simple liquids}

In physical chemistry, the van der Waals force (or van der Waals' interaction), is the sum of the attractive or repulsive forces between molecules (or between parts of the same molecule) other than those due to covalent bonds or the electrostatic interaction of ions with one another or with neutral molecules or charged molecules [11]. The term includes: force between two permanent dipoles (Keesom force), force between a permanent dipole and a corresponding induced dipole (Debye force) and force between instantaneously induced charge distributions (London dispersion force). The attractive London dispersion forces are the weakest forces. When considering "simple liquids", one normally refers to a system of particles with van der Waals' interactions, and the perturbation theories are often formulated for systems of spherically symmetrical particles and with only the first induced dipole-dipole term in the expansion of the attractive London dispersion force [8, 9]. The potential energy for such a system of $N$ spherical symmetrical particles in the volume $V$ (density $\rho=N / V$ ) and with interactions, given by a pair potential $u\left(r_{i j}\right)$ between particle No. $i$ and $j$ separated the distance $r_{i j}$, is as follows:

$$
U=\sum_{i<j}^{N} u\left(r_{i j}\right)
$$

and the equation of state is obtained by the virial equation for the pressure

$$
p=\rho T+p_{w}(\rho, T)=\rho T+\rho w(\rho, T),
$$

where the contribution to the pressure from the intermolecular forces, $p_{w}(\rho, T)$ is given by the value of the virial, $w(\rho, T)$

$$
w(\rho, T)=-\frac{2 \pi}{3} \rho \int_{0}^{\infty} g(r) \frac{\mathrm{d} u(r)}{\mathrm{d} r} r^{3} \mathrm{~d} r
$$

by integration over the radial distribution $g(r)$ of the virial between particles at the distance $r$.

The strength of the force $f(r)=-\frac{\mathrm{d} u(r)}{\mathrm{d} r}$ can be separated into short range force $f(r)$ for pair distances $r \leqslant \tilde{r}_{\mathrm{c}}$ and the long range force for $r>\tilde{r}_{\mathrm{c}}$. The separation between short- and long range forces, given by $\tilde{r}_{\mathrm{C}}$ is in the PT taken either to be where the potential $u(r)$ is zero [8] or where the force $f(r)$ is zero [9].

In PT, the mean distribution of the particles, $\tilde{g}(r)$ is obtained by taking only forces for $r \leqslant \tilde{r}_{\mathrm{c}}$ into account in the dynamics, and treating the virial contribution to the pressure for distances $r>\tilde{r}_{\mathrm{c}}$ as a mean field contribution, i.e., by assuming that

$$
\tilde{g}(r) \approx g(r) .
$$

The pressure is approximately obtained in the PT as follows:

$$
p(\rho, T) \approx \tilde{p}(\rho, T)+\Delta p(\rho, T)
$$


where

$$
\tilde{p}(\rho, T)=\rho T-\frac{2 \pi}{3} \rho^{2} \int_{0}^{\tilde{r}_{\mathrm{c}}} \tilde{g}(r) \frac{\mathrm{d} u(r)}{\mathrm{d} r} r^{3} \mathrm{~d} r
$$

is the pressure for a system with only short range forces for $r \leqslant \tilde{r}_{\mathrm{c}}$ and the value of the last term

$$
\Delta p(\rho, T)=-\frac{2 \pi}{3} \rho^{2} \int_{\tilde{r}_{\mathrm{c}}}^{\infty} \tilde{g}(r) \frac{\mathrm{d} u(r)}{\mathrm{d} r} r^{3} \mathrm{~d} r
$$

is obtained as a mean field term and using the the distribution $\tilde{g}(r)$ for a system with only short range interactions.

The pressure (and free energy) can be derived from the exact “ $\lambda$-expansion” [12]. The thermodynamic connection between any two systems with pair potential functions, $u(r)$ and $\tilde{u}(r)$ can be obtained from the pressure of a system with the potential

$$
u(r, \lambda)=\tilde{u}(r)+\lambda[u(r)-\tilde{u}(r)],
$$

where $u(r, \lambda)$ is equal to a reference potential $\tilde{u}(r)$ for $\lambda=0$ and equal to $u(r)$ for $\lambda=1$. The equilibrium pressures for systems with the pair potential $u(r, \lambda)$ and with values of $\lambda$ in the interval [0,1] establish a reversible path between the two systems, and the total differences in pressure, mean energy and free energy are then determined by numerical integration over different values of $\lambda$.

The second step in the PT is to relate $\tilde{p}(\rho, T)$ with the pressure $p_{0}\left(\rho_{0}\right)$ for a system of hard spheres with a diameter $r_{0}$ and corresponding density $\rho_{0}$, i.e., $\tilde{p}(\rho, T) \approx p_{0}\left(\rho_{0}, T\right)$. In this article we shall investigate only the validity of the first assumption in the PT, i.e., to what extent the structure of a simple liquid is given only by the forces $f(r)$ for $r \leqslant \tilde{r}_{\mathrm{c}}$.

\subsection{Separation of pair interactions into short- and long range interactions}

The effect of the attractive forces on the structure and dynamics is obtained for a system of particles with the standard Lennard-Jones (LJ) pair potential

$$
u_{\mathrm{LJ}}(r)=4 \varepsilon\left[(\sigma / r)^{12}-(\sigma / r)^{6}\right],
$$

and the equation of state and the diffusion are determined using Molecular Dynamics (MD) simulations. In MD, the interactions for distances $r_{\mathrm{c}} \gg \tilde{r}_{\mathrm{c}}$ are neglected. There are two standard ways to neglect these (very) long-range interactions. The usual way is to truncate and shift the potential, whereby the force, $f_{\mathrm{SP}}(r)$, is as follows:

$$
f_{\mathrm{SP}}(r)=\left\{\begin{array}{lll}
f_{\mathrm{LJ}}(r) & \text { if } & r<r_{\mathrm{c}} \\
0 & \text { if } & r>r_{\mathrm{c}}
\end{array}\right.
$$

This is referred to as a SP cut-off because it corresponds to shifting the potential below the cut-off and putting it to zero above which it ensures a continuity of the potential at $r_{\mathrm{c}}$ and avoids an infinite force herein. Another and more accurate way is to truncate and shift the forces (SF for shifted forces) [13] so that the force goes continuously to zero at $r_{\mathrm{c}}$, which is obtained by subtracting a constant term:

$$
f_{\mathrm{SF}}(r)=\left\{\begin{array}{lll}
f_{\mathrm{LJ}}(r)-f_{\mathrm{LJ}}\left(r_{\mathrm{c}}\right) & \text { if } & r<r_{\mathrm{c}} \\
0 & \text { if } & r>r_{\mathrm{c}}
\end{array}\right.
$$

This corresponds to the following modification of the potential [13]:

$$
u_{\mathrm{SF}}(r)=\left\{\begin{array}{lll}
u_{\mathrm{SF}}(r)=u_{\mathrm{LJ}}(r)-\left(r-r_{\mathrm{c}}\right) u_{\mathrm{LJ}}^{\prime}\left(r_{\mathrm{c}}\right)-u_{\mathrm{LJ}}\left(r_{\mathrm{c}}\right) & \text { if } & r<r_{\mathrm{c}} \\
0 & \text { if } & r>r_{\mathrm{c}}
\end{array}\right.
$$

The advance of the SF methods is that one can use any value of $\tilde{r}_{\mathrm{c}}$ or $r_{\mathrm{c}}$ without introducing discontinuities into the forces. Here, we shall use SF with $r_{\mathrm{c}}=4.5 \sigma$ as a good approximation for $r_{\infty}$. The maximum attraction of LJ forces at $r=(26 / 7)^{1 / 6} \sigma=1.244 \sigma$ is $f$ (max. att.) $=-2.3964 \epsilon / \sigma$ and the attraction at $r_{\mathrm{c}}=$ $4.5 \sigma$ is $f=-0.000642 \epsilon / \sigma$. The role of the attractions is obtained for the attractions in the interval $\left[\tilde{r}_{\mathrm{c}}, r_{\mathrm{c}}\right]$. The longer range attractions for $r \geqslant 4.5 \sigma$ contribute only marginally to the structure and dynamics except in very diluted gases (see later). 


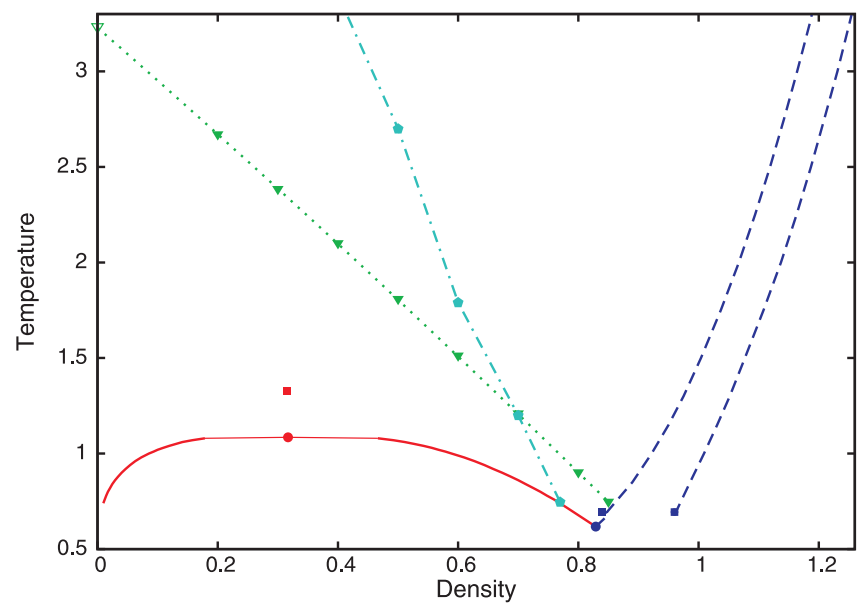

Figure 1. (Color online) The phase diagram $T(\rho)$ for a Lennard-Jones system. Red (solid) lines: coexisting liquid-gas [14] with critical point for $r_{\mathrm{C}}=2.5$ (red circle) [15] and (red square) [16] for $r_{\mathrm{C}}=\infty$; blue (dashed) lines coexisting solid-liquid lines [20] with triple point $\left(\rho_{\operatorname{tr}}(l), T_{\operatorname{tr}}\right)$ and $\left(\rho_{\operatorname{tr}}(s), T_{\operatorname{tr}}\right)$ [22] [23]; green (dotted) line: Zeno line (virial $=0$ ) and light blue (dash-dotted) line and points where excess energy and the virial (still) are strongly correlated $R=0.9$ [24].

\section{The effect of attractions on structure and pressure}

\subsection{The phase diagram of a LJ system}

The attractive forces can cause a condensation of particles in a gas state into liquid for sufficient low temperatures. The phase diagram $T(\rho)$ for a LJ system is shown in figure 国. The coexisting lines and points are all obtained by various MD simulations. There are several points to notice. The bimodals for coexisting gas and liquid [red (solid) lines] are sensitive to the value $r_{\mathrm{c}}$ for the truncation of the force field. The lines in the figure are for $r_{\mathrm{c}}=3 \sigma$ [14] connected with red short dashes to the critical point $\left(\rho_{\mathrm{c}}, T_{\mathrm{c}}\right)$, obtained by Smit [15] for $r_{\mathrm{c}}=2.5 \sigma$. Also shown in the figure (with a square) is the critical point $\left(\rho_{\mathrm{c}}, T_{\mathrm{c}}\right)=(0.316,1.326)$ for $r_{\mathrm{c}}=\infty$ obtained by Caillol [16]. The long range attractions behind a cut-off at $r_{\mathrm{c}}=2.5 \sigma$ significantly change the location of the critical point [16], as well as the surface tension and interfacial structure [17]. And for systems where the attractions are only short-ranged, there is no longer a critical point and a liquid condensation [18, 19].

The blue (dashed) lines in the figure [20] are the lines for the coexisting liquid and solid determined by extrapolations to $r_{\mathrm{c}}=r_{\infty}$. The location of the bimodals for coexisting liquid and solid (fcc) is less sensitive to the value of $r_{\mathrm{c}}$ [21], but the location of the triple point is also sensitive to the range of the attraction and is shifted from $T_{\mathrm{c}} \approx 0.69$ for $r_{\mathrm{c}}=r_{\infty}$ (blue square) [22] to $T_{\mathrm{c}}=0.618$ for $r_{\mathrm{c}}=2.5$ (blue circle) [23]. The light blue (dash-dotted) line and the points are the borderline where the excess potential energy and the virial still are strongly correlated [24,25]. The forces within the first coordination shell are dominant and ensure a strong correlation between the potential energy and the virial for state points to the right of this line [3].

\subsection{The zeno line}

Green full triangles in figure 1 are points on the "zeno line" 26] where the pressure equals the pressure of an ideal gas. The points are fitted with a second order polynomial (green line) and the zeno line is weakly curved. All points at the zeno line are obtained for SF with $r_{\mathrm{C}}=4.5 \sigma$. The point $\left(0, T_{\mathrm{B}}\right)$ with the Boyle temperature $T_{\mathrm{B}}=3.2310$ is obtained from the expression for the second virial coefficient. The Boyle temperature for a LJ system $\left(r_{\mathrm{C}}=\infty\right)$ is $T_{\mathrm{B}}=3.418$, and the value of the Boyle temperature for $r_{\mathrm{C}}=4.5 \sigma$ is $T_{\mathrm{B}}=3.2310$. It shows that long-range attractive forces behind $r_{\mathrm{C}}=4.5 \sigma$ play a role in (very) diluted gases.

\footnotetext{
${ }^{1}$ Units: length in unit of $\sigma$; time in units of $\sigma \sqrt{\epsilon / m}$; energy in units of $\epsilon / k_{\mathrm{B}}$.
} 
The virial, $w$ and the contribution $\rho w$ to the pressure is zero for state points at the zeno line. The contribution $p_{w}=\rho w$ to the pressure from the forces can be divided into contributions from the repulsive and the attractive forces

$$
\begin{aligned}
& p_{w}(+)=\rho w(+)=-\frac{2 \pi}{3} \rho^{2} \int_{0}^{r_{\mathrm{WCA}}} g(r) \frac{\mathrm{d} u(r)}{\mathrm{d} r} r^{3} \mathrm{~d} r>0, \\
& p_{w}(-)=\rho w(-)=-\frac{2 \pi}{3} \rho^{2} \int_{r_{\mathrm{WCA}}}^{\infty} g(r) \frac{\mathrm{d} u(r)}{\mathrm{d} r} r^{3} \mathrm{~d} r<0,
\end{aligned}
$$

where $r_{\mathrm{WCA}}=2^{1 / 6}$ for a LJ system. Hence, the zeno line is a borderline between the dominance of the repulsive and the attractive forces on the pressure. For state points above the line, the effect on the pressure from the virial of the repulsive forces is dominant. The structure of the fluid, given by the radial distribution function ensures a positive value of the the virial, i.e. $p_{w}(+)>-p_{w}(-)$, and visa verse for state points below the line.

The central assumption in PT is that the structure is given by the repulsive forces, and thus it is natural to investigate whether it is the case for state points along the zeno line, where the contributions to the virial from the repulsive and the attractive forces cancel. The LJ potential with only the first term in the induced multipole interactions is the potential for the weakest London forces, and all real (simple) van der Waals liquids have stronger attractions than a LJ system.

\subsection{The structure of the fluid at the zeno line}

The impact of the attractive forces on the structure of the fluid can be determined by obtaining the structure of the fluid with and without the attraction included in the dynamics. The attractive force changes the distributions of particles. Figure 2 and figure 3 show the radial distribution functions for the condensed liquid state $(\rho, T)=(0.85,0.7453)$ and for the diluted gas state $(\rho, T)=(0.20,2.6680)$, respectively. Both state points are on the zeno line, and the effect of the attractive forces on the structure is bigger for the points below this line. The black (solid) line in figure 2 is the distribution $g(r)$ for a LJ fluid, and the red (dashed) line is the distribution $\tilde{g}(r)$ when only forces within the FCS are included in the dynamics. The green (dotted) line is $\tilde{g}(r)$ for $\tilde{r}_{\mathrm{c}}=2^{1 / 6}$, where only the repulsive forces are included in the dynamics. The inset shows the corresponding differences $\tilde{g}(r)-g(r)$. For this condensed fluid, the attractive forces behind the FCS have only a marginal effect on the distribution, but the attractive forces within the FCS change the structure of the fluid significantly [3].

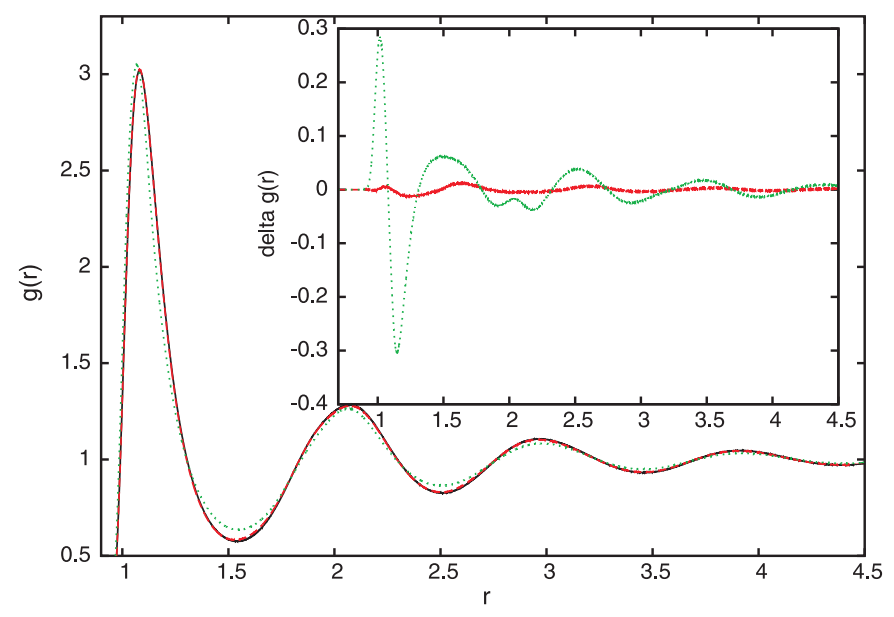

Figure 2. (Color online) Radial distribution function, $g(r)$, for $\rho=0.85$ and $T=0.7453$ (at the zeno line). Black (solid): $\mathrm{g}(\mathrm{r})$; red (dashed): $\tilde{g}(r)$ for $\tilde{r}_{\mathrm{C}}=1.54$ (first coordination shell) and green (dotted): $\tilde{r}_{\mathrm{C}}=2^{1 / 6}$ (WCA). The inset show the difference, $\tilde{g}(r)-g(r)$. 


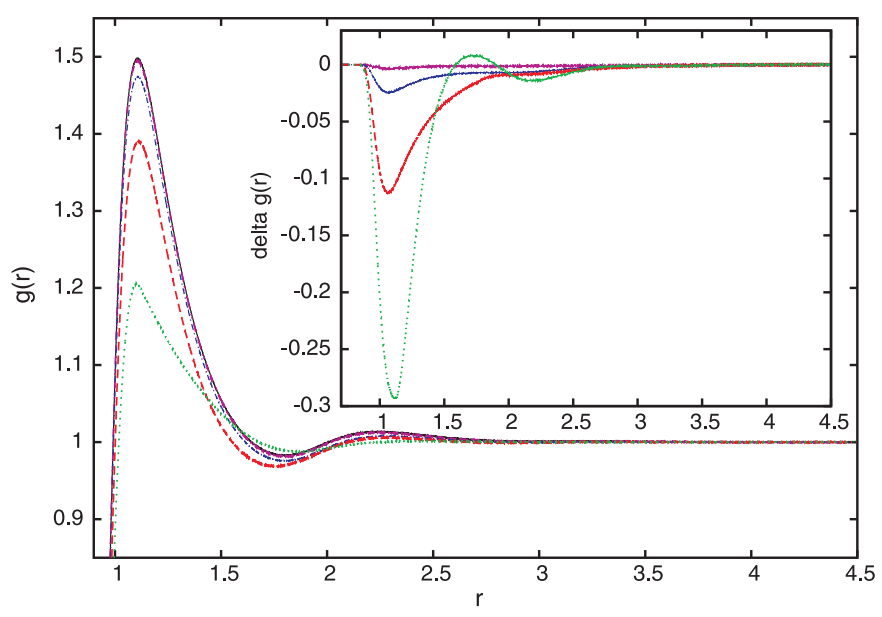

Figure 3. (Color online) Radial distribution function, $g(r)$, for $\rho=0.20$ and $T=2.668$ (at the zeno line). Black (solid): $\mathrm{g}(\mathrm{r})$; red (dashed): $\tilde{g}(r)$ for $\tilde{r}_{\mathrm{C}}=1.75$ (first coordination shell) and green (dotted): $\tilde{r}_{\mathrm{C}}=2^{1 / 6}$ (WCA). Also shown is the distribution for $\tilde{r}_{\mathrm{C}}=2.5$ in blue (dash-dotted) and $\tilde{r}_{\mathrm{C}}=3.5$ in magenta (dashdouble-dotted). The inset show the differences, $\tilde{g}(r)-g(r)$.

Figure 3 shows the corresponding distributions at the diluted state with $\rho=0.2$. Also included [with blue (dash-dotted)] is the distribution when the effect of the forces from the second coordination shell is included in the dynamics, and the magenta (dash-double-dotted) curve is for $\tilde{r}_{\mathrm{c}}=3.5$. The two figures demonstrate that nowhere on the zeno line there is a structure of the fluid given only by the repulsive forces. The insets show that the main differences in the distributions appear for distances within the FCS, and that the differences are of the same order for the two states. The distributions with blue (dashdotted) and magenta (dash-double-dotted) in figure 3 show that whereas at condensed states it is the forces within the FCS which change the structure compared to the structure of a repulsive system, the longer range attractions behind the FCS also have an effect on the structure of a more diluted fluid.

\subsection{Pressure along the zeno line}

According to figure 2 and figure 3 the radial distribution $\tilde{g}(r)$ differs from $g(r)$ if the attractive forces are neglected in the dynamics. The integral effect on the pressure is given by

$$
\begin{aligned}
& \tilde{p}_{w}(+)=\rho \tilde{w}(+)=-\frac{2 \pi}{3} \rho^{2} \int_{0}^{r \text { WCA }} \tilde{g}(r) \frac{\mathrm{d} u(r)}{\mathrm{d} r} r^{3} \mathrm{~d} r, \\
& \tilde{p}_{w}(-)=\rho \tilde{w}(-)=-\frac{2 \pi}{3} \rho^{2} \int_{r \text { WCA }}^{\infty} \tilde{g}(r) \frac{\mathrm{d} u(r)}{\mathrm{d} r} r^{3} \mathrm{~d} r .
\end{aligned}
$$

The two contributions and their sum are shown in figure 4 With black (solid) is $p_{w}, p_{w}(+)$ and $p_{w}(-)$ for a LJ fluid, with red (dashed) $\tilde{p}_{w}, \tilde{p}_{w}(+)$ and $\tilde{p}_{w}(-)$ for $\tilde{g}(r)$ with forces only from the FCS. The green (dotted) curves are $\tilde{p}_{w}, \tilde{p}_{w}(+)$ and $\tilde{p}_{w}(-)$ for a system with only repulsive LJ forces. Distributions are changed by neglecting the attractive forces in the dynamics, but the integral effect of the attractions on the pressure, $\tilde{p}_{w}(-)$ is close to zero. This is, however, not the case for the effect on the pressure $\tilde{p}_{w}(+)$ from changes in the distribution for distances where the forces are repulsive. Figure 4 reveals that the pressure obtained by PT fails, not at diluted supercritical states, but for condensed states $\rho>0.6$, and it fails because the structure of the fluid for distances where the forces are repulsive is changed due to the effect of the attractive forces! Figure 2 shows [green (dotted) lines] that the radial distribution function $\tilde{g}(r)$ for $r<2^{1 / 6}$ is "parallel shifted" toward shorter distances by neglecting the attraction in the dynamics, and it is this effect which leads to the deviation in the virial and pressure $\tilde{p}_{w}(+)-p_{w}(+)$ for a condensed fluid. 


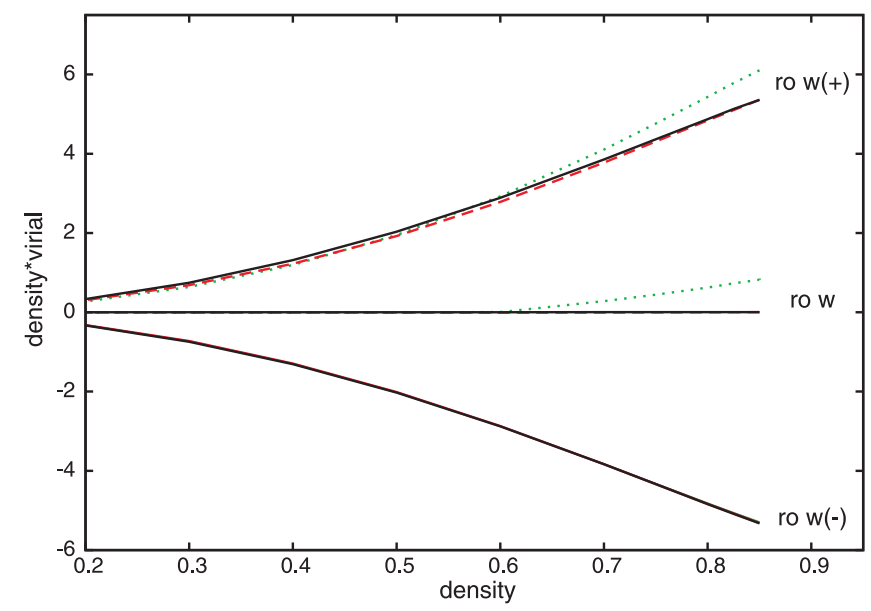

Figure 4. (Color online) Contribution to the pressure from the virial, $p_{w}=\rho w$, along the zeno line. The contributions are divided into the contribution from the repulsive forces, $\rho w(+)$ and the attractive forces, $\rho w(-)$. With black (solid) is the two contributions and their sum (which is equal to zero), and with red (dashed) are the corresponding contributions obtained from $\tilde{g}(r)$ by neglecting the attractive forces behind the first coordination shell in the dynamics. Green (dotted) are obtained from $\tilde{g}(r)$ for a system of repulsive LJ particles.

The apparent agreement between the radial distribution function of a LJ system and the corresponding distribution for a system with only repulsive forces for a liquid is striking (figure 2); but there are systematic differences at short distances and these differences lead to a too high pressure. The standard perturbation theory (PT) (Barker-Henderson [8] and Weeks-Chandler-Anderson [9]) is not quantitatively correct. The central assumption in PT is that the effect of the attraction can be obtained from a fluid with only repulsive forces. It is, however, possible to modify the PT by replacing the reference system with a reference system with the forces within the first coordination shell [3]. Doing so, the pressure obtained by PT is not only qualitatively and to a high extent also quantitatively correct for diluted gas and super critical states as well as for condensed liquid states.

The standard perturbation theory is the "zero" term in an exact $\lambda$ expansion [12], and it is, of course, possible to obtain the pressure from the pressure $\tilde{p}(\rho, T)$ for the short range potential by determining the distribution for the potential $u(r, \lambda)$ for sufficient values of $\lambda$ [27, 28].

\section{The role of attractions on self-diffusion}

The attractive forces change the dynamics of a system. The effect of the attractive forces on the selfdiffusion coefficient (SDC) is determined for the critical isotherm $T=T_{\mathrm{c}}$ and the triple point fluid isochore $\rho_{\text {tr }}$ for a LJ system. The values of the SDC for the critical LJ isotherm $T_{\mathrm{c}}(\mathrm{LJ})=1.326$ are shown in figure 5 The values are obtained from the mean displacements of the $N=2000$ particles and for $1.6 \times 10^{6}$ time steps with $\delta t=0.0025$. For the system with only short-range attractions [green (dotted) line], the temperature is well above its critical temperature, and the system with no attractions [blue (dashed) line] has no critical temperature. The SDC for all three systems decline exponentially with the density in the interval $\rho \in[0.2,0.8]$ (inset). For densities $\rho>0.5$, there are no significant differences between the coefficients for the LJ system [red (solid) line] and the coefficients for the system with only forces within the FCS in the dynamics [green (dotted) line] [13], but for smaller densities also the longer ranged forces play a role and damp the diffusion. The diffusion in a LJ system is systematically smaller than the diffusion for a system with only repulsive forces [red (solid) and blue (dashed) lines], and the differences increase with decreasing density.

There is no observable change in the behavior of the SDC of the LJ system near the critical point. Only the LJ system exhibits critical fluctuations. The system with only short-range attractions from the FCS 


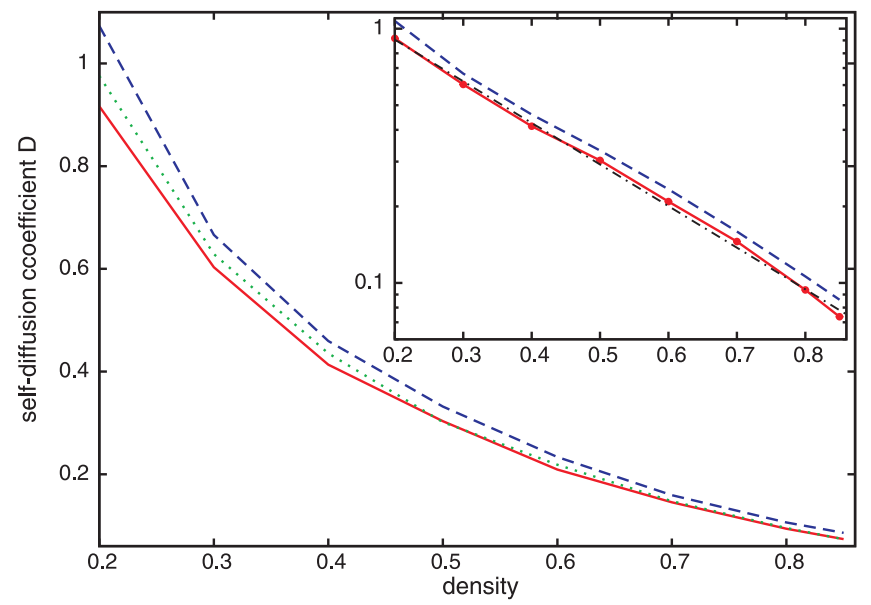

Figure 5. (Color online) Self-diffusion coefficients $D(T, \rho)$ for the critical $\mathrm{LJ}$ isotherm $T=T_{\mathrm{C}}=1.326$. Red (solid) line: LJ fluid; green (dotted): for a system with only forces within the FCS included in the dynamics, and blue (dashed) is when only the repulsive forces are included in the dynamics. The inset shows $\ln D(T, \rho)$ and with black (dash-dotted): $a \exp (-b \rho)$.

has a lower critical temperature, and the system with only repulsive forces has no critical temperature. Nevertheless, the three systems exhibit a coherent behavior and with no spur of a critical effect, which should be in only one of the three systems. The critical point marks the location of a thermodynamic instability and the effect on the SDC is small, if any, and in agreement with experimental data [29-31].

The SDC varies exponentially with increasing density. The inset shows $\log D$ for the LJ system [red (solid)] and the system without attractive forces [blue (dashed)]. The coefficients $a$ and $b$ in the function $a \mathrm{e}^{-b \rho}$ are fitted to the values of $D$ for the LJ system, and the function is shown in the inset [black (dash-dotted) straight line]. The data show that the role of the attraction is given by the value of the preexponential coefficient $a$, whereas the values of the coefficient $b$ for the two systems are the same within the accuracy of the MD simulation.

The SDC are usually compared with the coefficients obtained using a modified Cohen-Turnbull theory [32, 33]. In this theory, the diffusion coefficients are given by

$$
D=D_{0} \exp \left(-\alpha \frac{v_{\mathrm{c}}}{v_{\mathrm{f}}}\right),
$$

where $D_{0}$ is the Chapman-Enskog diffusion for a model pair potential at low density

$$
D_{0}=D_{0}^{\mathrm{HS}} / \Omega^{(1,1)}(T) .
$$

The coefficient $D_{0}^{\mathrm{HS}}$ is the SDC for a hard sphere system, the temperature dependent collision integral, $\Omega^{(1,1)}(T)$, depends on the attractive forces [34], $\alpha$ is an ("overlap") parameter, $v_{\mathrm{c}}$ is "the critical volume" and $v_{\mathrm{f}}$ is the "mean free volume". The data in figure 5 agree with this model with $a=D_{0}, b=\alpha v_{\mathrm{c}}$ and $\nu_{\mathrm{f}} \approx \rho^{-1}$.

The SDC for the triple point (fluid) density $\rho_{\text {tr }}=0.85$ are shown in figure 6. With red (solid) line and points are $D$ for the LJ system. The triple point temperature is $T_{\text {tr }}=0.69$ and the system is supercooled down below $T_{\text {tr }}$ to $T=0.30$ where it crystallizes. The diffusion coefficients for the system with only shortrange attraction (green) are almost equal to the diffusion coefficients for the LJ system, showing that the long range attractions behind the FCS only have a marginal effect on the diffusion at high temperatures as well as on the diffusion at super-cooled temperatures. The diffusion coefficients for the system without attractive forces [blue (dashed) line and points] are, however, systematically higher. The SDC for the three systems are all given by a simple linear dependence $a+b T$ where $b \approx$ independent of the range of the attractive forces, and $a$ 's dependence on the attractive forces to a high accuracy is given by the short range forces within the FCS. At a deeper super cooling for a system which is more resistant against 


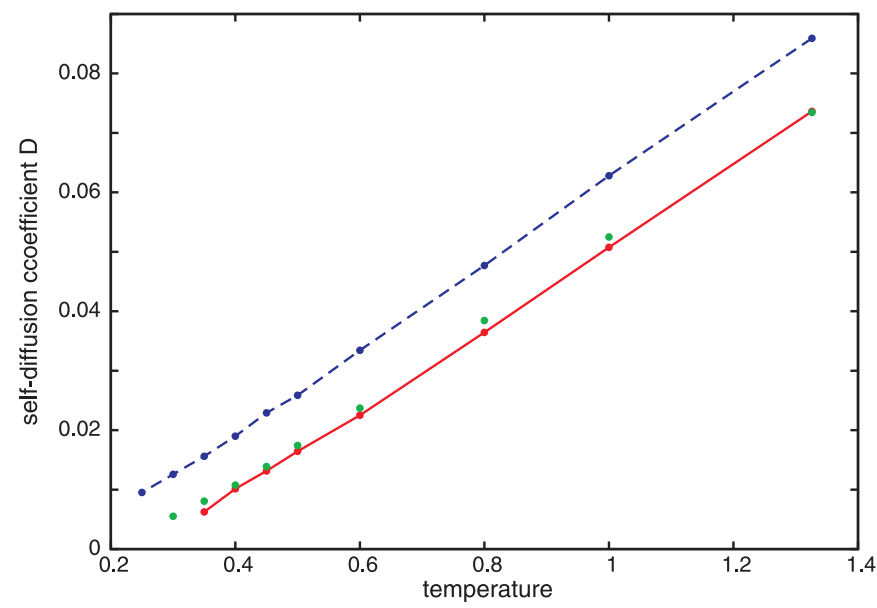

Figure 6. (Color online) Self-diffusion coefficients $D(T, \rho)$ for the triple point fluid density $\rho_{\operatorname{tr}}=0.85$. Red (solid) line and points: LJ fluid; green points: for a system with only forces within the FCS included in the dynamics, and blue (dashed) line and points are when only the repulsive forces are included in the dynamics.

crystallization [35, 36], the dynamics and the SDC can still be obtained with only attractive forces within the FCS included in the dynamics [3], whereas the SDC for a system with only repulsive forces deviates significantly [3, 37].

\section{Conclusion}

Liquids are often separated into simple van der Waals liquids with weak intermolecular attractive forces and into liquids with stronger attractions. The van der Waals theory for simple liquids, where the repulsive and the weak attractive forces give rise to separate modifications of volume and pressure in the ideal-gas equation of state, is qualitatively correct and has played a historical role in understanding the fluid systems. The perturbation theories (PT) for simple liquids, which were formulated late in the twentieth century, showed good agreements with experimental- and computer simulated data, and gave reason to believe that it is indeed possible to obtain the contribution to the pressure from the weak attractive van der Waals forces as a mean field contribution from a system with the particle distribution for a system with only strong repulsive forces. Since then, the development in computer simulations has made it possible to obtain the equation of state for systems with different strength of attractions with high accuracy, and to investigate various approximations in the PT theories for fluid systems. The present investigation shows that, even for a system with the most weak attractive London forces, nowhere in the phase space, it is possible to approximate the radial distribution of particles with the distribution obtained for a system with only repulsive forces. Even weak attractive induced dipole forces, primarily within the first coordination shell, modify the radial distribution, especially at short intermolecular distances (figure2), and with the result that the pressure obtained by the PT is too high (figure 4 .

The weak long-range attractions damp the dynamics and the diffusion (figure5 5 and figure 6). Since it is the attractive forces which cause a condensation into a liquid for temperatures below the critical temperature, $T_{\mathrm{c}}$, one should a priory assume that the attractive forces, which cause long range fluctuations in densities at $T_{\mathrm{c}}$ would affect the particle diffusion and the value of the self-diffusion constant (SDC) near the critical point. However, this is not the case, and the result is in accordance with experimental data for the SDC in the critical region [31].

The SDC along the critical isotherm exponentially varies with the density (figure 5). This is in good agreement with a modified Cohen-Turnbull model for the SDC [32, 33]. The effect of the attractive forces on the SDC is a simple damping of the diffusion and with a reduced value of the preexponential constant. The attractive forces also damp the diffusion in condensed super-cooled fluids. The temperature depen- 
dence of the SDC at the triple-point isochore shows a simple linear damping with respect to temperature, and the damping of the diffusion is caused by the attractive forces within the first coordination shell (figure 6). For systems which are less prone to crystallization and at a deeper super-cooling, the effect of the attractive forces is more dominant, but the attractive forces within the FCS still determine the diffusion and viscosity, as well as the thermodynamics [3].

\section{Acknowledgements}

The centre for viscous liquid dynamics "Glass and Time” is sponsored by the Danish National Research Foundation (DNRF).

\section{References}

1. Van der Waals J.D., On the Continuity of the Gaseous and Liquid States, Thesis, Universiteit Leiden, 1873.

2. Rowlinson J.S., Widom B., Molecular Theory of Capillarity, Claredon Press, Oxford, 1982, Chapter 1.6.

3. Toxvaerd S., Dyre J.C., J. Chem. Phys., 2011, 135, 134501; doi 10.1063/1.3643123

4. Widom B., Science, 1967, 157, 375; doi 10.1126/science.157.3787.375

5. Widom B., J. Chem. Phys., 1965, 43, 3898; doi 10.1063/1.1696618.

6. Zwanzig R.W., J. Chem. Phys., 1954, 22, 1420; doi 10.1063/1.1740409

7. Longuet-Higgens H.C., Widom B., Mol. Phys., 1964, 8, 549; doi 10.1080/00268976400100611

8. Barker J.A., Henderson D., J. Chem. Phys., 1967, 47, 4714; doi 10.1063/1.1701689

9. Weeks J., Chandler D., Andersen H.C., J. Chem. Phys., 1971, 54, 5237; doi 10.1063/1.1674820

10. Barker J.A., Henderson D., Rev. Mod. Phys., 1976, 48, 587; doi 10.1103/RevModPhys.48.587

11. Compendium of Chemical Terminology - IUPAC Recommendations, 2nd ed. (the "Gold Book"), McNaught A.D., Wilkinson A. (Eds.), Blackwell Scientific Publications, Oxford, 1997 [Online corrected version: "van der Waals forces", 1994].

12. Hansen J.-P., McDonald I.R., Theory of Simple Liquids, Academic Press, London, 1990, Chapter 6.2.

13. Toxvaerd S., Dyre J.C., J. Chem. Phys., 2011, 134, 081102; doi 10.1063/1.3558787

14. Watanabe H., Ito N., Hu C.-K., J. Chem. Phys., 2012, 136, 204102; doi 10.1063/1.4720089

15. Smit B., J. Chem. Phys., 1992, 96, 8639; doi 10.1063/1.462271

16. Caillol J.M., J. Chem. Phys., 1998, 109, 4885; doi 10.1063/1.477099

17. Grosfils P., Lutsko J.F., J. Chem. Phys., 2009, 130, 054703; doi 10.1063/1.3072156

18. Hagen M.H.J., Frenkel D., J. Chem. Phys., 1994, 101, 4093; doi 10.1063/1.467526

19. Vliegenthart G.A., Lekkerkerker H.N.W., J. Chem. Phys., 2000, 112, 5364; doi $10.1063 / 1.481106$

20. Barroso M.A., Ferreira A.L., J. Chem. Phys., 2002, 116, 7145; doi $10.1063 / 1.1464828$

21. Mastny E.A., de Pablo J.J., J. Chem. Phys., 2007, 127, 104504; doi 10.1063/1.2753149

22. Hansen J.-P., Verlet L., Phys. Rev., 1969, 184, 151; doi 10.1103/PhysRev.184.151

23. Toxvaerd S., J. Phys. Chem. C, 2007, 111, 15620; doi 10.1021/jp073665x

24. Bailey N.P., Pedersen U.R., Gnan N., Schrøder T.B., Dyre J.C., J. Chem. Phys., 2008, 129, 184507; doi $10.1063 / 1.2982247$

25. Gnan N., Schrøder T.B., Pedersen U.R., Bailey N.P., Dyre J.C., J. Chem. Phys., 2009, 131, 234504; doi $10.1063 / 1.3265957$

26. Ben-Amotz D., Herschbach D.R., Isr. J. Chem., 1990, 30, 59; doi 10.1002/ijch.199000007

27. Weeks J.D., Broughton J.Q., J. Chem. Phys., 1983, 78, 4197; doi 10.1063/1.445097

28. Rull L.F., Cuadros F., Morales J.J., Phys. Rev. A, 1984, 30, 2781; doi 10.1103/PhysRevA.30.2781

29. Drozdov A.N., Tucker S.C., J. Chem. Phys., 2002, 114, 4912; doi 10.1063/1.1349095

30. Drozdov A.N., Tucker S.C., J. Chem. Phys., 2002, 116, 6381; doi 10.1063/1.1458929

31. Harris K.R., J. Chem. Phys., 2002, 116, 6379; doi 10.1063/1.1458928

32. Laghaei R., Nasrabad A.E., Eu B.C., J. Phys. Chem. B, 2005, 109, 21375; doi 10.1021/jp050504p

33. Nasrabad A.E., J. Chem. Phys., 2008, 129, 244508; doi 10.1063/1.3041421

34. Hirschfelder J.O., Curtiss C.F., Bird R.B., Molecular Theory of Gases and Liquids, Wiley \& Sons, New York, 1954, Chapter 7.

35. Kob W., Andersen H.C., Phys. Rev. E, 1995, 51, 4626; doi 10.1103/PhysRevE.51.4626

36. Kob W., Andersen H.C., Phys. Rev. E, 1995, 52, 4134; doi 10.1103/PhysRevE.52.4134

37. Berthier L., Tarjus G., Phys. Rev. Lett., 2009, 103, 170601; doi 10.1103/PhysRevLett.103.170601 


\title{
Роль сил притягання у визначенні рівноважної структури і динаміки простих рідин
}

\section{С. Токсваерд}

Факультет природничих наук, Університет м. Роскільде, DK-4000 Роскільде, Данія

\begin{abstract}
Моделювання методом молекулярної динаміки системи Лєннарда-Джонса з різною областю притягання показують, що сили притягання видозміюють радіальний розподіл частинок. Сили в межах першої координаційної сфери (ПКС) є важливими лише для густих рідин, в той час, як для газів і помірно густих плинів, навіть сили притягання за межами ПКС відіграють певну роль. Зміни у розподілі, спричинені нехтуванням силами притягання, приводять до занадто високого тиску. Слабке далекосяжне притягання гасить динаміку і дифузію частинок у станах газу, надкритичного плину та рідини. Значення коефіцієнтів самодифузії якісно узгоджуються з модифікованою моделлю Коена-Турнбулла. Коефіцієнти самодифузії вздовж критичної ізотерми не показують ніякої аномалії у критичній точці, що узгоджується з експериментальними даними.
\end{abstract}

Ключові слова: прості рідини, рівняння стану, теорія збурень, самодифузія 


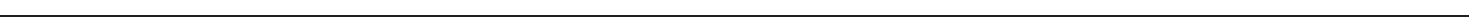

